\title{
AOMOTO'S MACHINE AND THE Dyson CONSTANT TERM IDENTITY
}

\author{
Kevin W. J. Kadell
}

\begin{abstract}
Aomoto has used the fundamental theorem of calculus to give an elegant proof of an extension of Selberg's integral. A constant term formulation of Aomoto's argument is based upon the fact that for $1 \leq s \leq n$, the constant term in $t_{s} \partial / \partial t_{s} f\left(t_{1}, \ldots, t_{n}\right)$ is zero provided that $f\left(t_{1}, \ldots, t_{n}\right)$ has a Laurent expansion around $t_{1}=\cdots=t_{n}=0$. We use this as the engine for a simple proof of an Aomoto-type extension of the Dyson constant term identity. We outline the use of Good's proof to evaluate the coefficients of $t_{1} / t_{n}, t_{1} t_{2} / t_{n-1} t_{n}$, and $t_{1} t_{2} / t_{n}^{2}$ in $\prod_{1 \leq i<j \leq n}\left(1-t_{i} / t_{j}\right)^{a_{i}}\left(1-t_{j} / t_{i}\right)^{a_{j}}$. We give a conjecture with some surprising symmetries and its $q$-analogue.
\end{abstract}

\section{Introduction and summary}

Selberg [14] has given an important multivariable beta integral which is related to constant term identities associated with root systems. Aomoto [2] has extended Selberg's integral to

$$
\begin{array}{r}
\int_{0}^{1} \ldots \int_{0}^{1} \prod_{i=1}^{n} t_{i}^{(x-1)+\chi(i \leq m)}\left(1-t_{i}\right)^{(y-1)} \Delta_{n}^{2 k}\left(t_{1}, \ldots, t_{n}\right) d t_{1} \cdots d t_{n} \\
=\prod_{i=1}^{n} \frac{\Gamma(x+(n-i) k+\chi(i \leq m)) \Gamma(y+(n-i) k)}{\Gamma(x+y+(2 n-i-1) k+\chi(i \leq m)} \frac{\Gamma(1+i k)}{\Gamma(1+k)}
\end{array}
$$

where $n, k$, and $m$ are integers with $n \geq 1, k \geq 0$, and $0 \leq m \leq n, \operatorname{Re}(x)>0$, $\operatorname{Re}(y)>0, \chi(A)$ is one or zero according to whether $A$ is true or false, respectively, and

$$
\Delta_{n}\left(t_{1}, \ldots, t_{n}\right)=\prod_{1 \leq i<j \leq n}\left(t_{i}-t_{j}\right)
$$

denotes the Vandermonde determinant. When $m=0,(1.2)$ is Selberg's integral in which the integrand is symmetric in $t_{1}, \ldots, t_{n}$. Observe that the effect of the parameter $m$ is to introduce the product $t_{1} \cdots t_{m}$ into the integrand.

Aomoto's elegant proof [2] is based upon the fact that if $f\left(t_{1}, \ldots, t_{n}\right)$ is continuous on the unit cube $[0,1]^{n}$, then, by the fundamental theorem of calculus, we have

$$
0=\int_{0}^{1} \cdots \int_{0}^{1} \frac{\partial}{\partial t_{1}}\left(t_{1}\left(1-t_{1}\right) f\left(t_{1}, \ldots, t_{n}\right)\right) d t_{1} \cdots d t_{n} .
$$

Received March 31, 1998, revised May 21, 1998.

1991 Mathematics Subject Classification: 33C70, 33C80, 33D70, 33D80, 05A15, $05 \mathrm{E} 99$.

Key words and phrases: Dyson's constant term identity, Selberg's integral, Aomoto's machine. 
Morris [13] has proven the case $m=0$ of the following constant term identity which is equivalent to Aomoto's integral (1.1):

$$
\begin{gathered}
\text { [1] } \prod_{i=1}^{n}\left(1-t_{i}\right)^{a+\chi(i \leq m)}\left(1-\frac{1}{t_{i}}\right)^{b} \prod_{1 \leq i<j \leq n}\left(1-\frac{t_{i}}{t_{j}}\right)^{k}\left(1-\frac{t_{j}}{t_{i}}\right)^{k} \\
=\prod_{i=1}^{n} \frac{(a+b+(n-i) k+\chi(i \leq m)) !}{(a+(n-i) k+\chi(i \leq m)) !(b+(n-i) k) !} \frac{(i k) !}{k !}
\end{gathered}
$$

where $[w] f$ denotes the coefficient of the monomial $w$ in the Laurent expansion of $f$.

Let $1 \leq s \leq n$. Aomoto's argument may be applied to constant term identities by observing that if $f\left(t_{1}, \ldots, t_{n}\right)$ has a Laurent expansion around $t_{1}=\cdots=t_{n}=0$, then we have

$$
0=[1] t_{s} \frac{\partial}{\partial t_{s}}\left(f\left(t_{1}, \ldots, t_{n}\right)\right)
$$

Let $n \geq 2$ and $a_{1}, \ldots, a_{n} \geq 0$. We set

$$
f_{n}\left(a_{1}, \ldots, a_{n} ; t_{1}, \ldots, t_{n}\right)=\prod_{1 \leq i<j \leq n}\left(1-\frac{t_{i}}{t_{j}}\right)^{a_{i}}\left(1-\frac{t_{j}}{t_{i}}\right)^{a_{j}}
$$

and use capital letters to denote the constant term

$$
F_{n}\left(a_{1}, \ldots, a_{n}\right)=[1] f_{n}\left(a_{1}, \ldots, a_{n} ; t_{1}, \ldots, t_{n}\right) .
$$

Dyson [4] conjectured the constant term identity

$$
F_{n}\left(a_{1}, \ldots, a_{n}\right)=\frac{\left(a_{1}+\cdots+a_{n}\right) !}{a_{1} ! \cdots a_{n} !},
$$

which was proven independently by Gunson [6] and Wilson [16]. Good [5] gave a short proof of (1.8) using the identity

$$
1=\sum_{j=1}^{n} \prod_{\substack{i=1 \\ i \neq j}}^{n}\left(1-\frac{t_{j}}{t_{i}}\right)^{-1}
$$

which may be obtained [7] by expanding the Vandermonde determinant

$$
\Delta_{n}\left(t_{1}, \ldots, t_{n}\right)=\operatorname{det}\left|t_{j}^{n-i}\right|_{n \times n}
$$

along the bottom row.

Observe that Good's identity (1.9) gives

$$
f_{n}\left(a_{1}, \ldots, a_{n} ; t_{1}, \ldots, t_{n}\right)=\sum_{j=1}^{n} f_{n}\left(a_{1}, \ldots, a_{j-1}, a_{j}-1, a_{j+1}, \ldots, a_{n} ; t_{1}, \ldots, t_{n}\right) .
$$

Extracting the constant term from (1.11), we have

$$
F_{n}\left(a_{1}, \ldots, a_{n}\right)=\sum_{j=1}^{n} F_{n}\left(a_{1}, \ldots, a_{j-1}, a_{j}-1, a_{j+1}, \ldots, a_{n}\right) .
$$


Good's proof [5] of the Dyson constant term identity (1.8) is concluded by observing that the multinomial coefficient $\left(a_{1}+\cdots+a_{n}\right) ! / a_{1} ! \cdots a_{n}$ ! also satisfies $(1.12)$ and the boundary condition

$$
F_{n}\left(a_{1}, \ldots, a_{m-1}, 0, a_{m+1}, \ldots, a_{n}\right)=F_{n-1}\left(a_{1}, \ldots, a_{m-1}, a_{m+1}, \ldots, a_{n}\right) .
$$

Let $[1, n]=\{1, \ldots, n\}$ denote the set of positive integers from one to $n$. We set

$$
\begin{aligned}
k_{n, m}\left(a_{1}, \ldots, a_{n} ; t_{1}, \ldots, t_{n}\right)= & \sum_{p=1}^{n} \sum_{\substack{M \subseteq[1, n]-\{p\} \\
|M|=m}}\left(1+\sum_{v \in[1, n]-M} a_{v}\right) \\
& \times \prod_{s \in M}\left(1-\frac{t_{p}}{t_{s}}\right) f_{n}\left(a_{1}, \ldots, a_{n} ; t_{1}, \ldots, t_{n}\right),
\end{aligned}
$$

and use capital letters to denote the constant term

$$
K_{n, m}\left(a_{1}, \ldots, a_{n}\right)=[1] k_{n, m}\left(a_{1}, \ldots, a_{n} ; t_{1}, \ldots, t_{n}\right) .
$$

Observe that we may relax the restriction $M \subseteq[1, n]-\{p\}$ to $M \subseteq[1, n]$ since $p \in M$ implies that $\prod_{s \in M}\left(1-t_{p} / t_{s}\right)=0$. It will be convenient for the proof not to do so.

We use (1.5) as the engine for a simple proof of an Aomoto-type generalization of the Dyson constant term identity (1.8). This is given by the following theorem which is our main result.

\section{Theorem 1.}

$$
K_{n, m}\left(a_{1}, \ldots, a_{n}\right)=n\left(\begin{array}{c}
n-1 \\
m
\end{array}\right)\left(1+\sum_{v=1}^{n} a_{v}\right) \frac{\left(a_{1}+\cdots+a_{n}\right) !}{a_{1} ! \cdots a_{n} !} .
$$

Let $\pi \in S_{n}$. The case where $\pi$ is a transposition of the symmetry

$$
f_{n}\left(a_{\pi(1)}, \ldots, a_{\pi(n)} ; t_{\pi(1)}, \ldots, t_{\pi(n)}\right)=f_{n}\left(a_{1}, \ldots, a_{n} ; t_{1}, \ldots, t_{n}\right)
$$

plays a silent role in our proof of Theorem 1 . We avoid its use by using the engine (1.5) with $s$ ranging from one to $n$ and combining terms so as to remove the denominators from the partial derivatives which arise when we use the engine (1.5) of our machine.

Defining the partial $q$-derivative by

$$
\frac{\partial_{q}}{\partial_{q} t_{s}}\left(f\left(t_{1}, \ldots, t_{n}\right)\right)=\frac{\left(f\left(t_{1}, \ldots, t_{n}\right)-f\left(t_{1}, \ldots, t_{s-1}, q t_{s}, t_{s+1} \ldots, t_{n}\right)\right)}{t_{s}(1-q)}
$$

we see that if $f\left(t_{1}, \ldots, t_{n}\right)$ has a Laurent expansion around $t_{1}=\cdots=t_{n}=0$, then we have

$$
0=[1] t_{s} \frac{\partial_{q}}{\partial_{q} t_{s}}\left(f\left(t_{1}, \ldots, t_{n}\right)\right) .
$$

Observe that the $q$-engine (1.19) expresses the fact that the constant term is unchanged by the substitution $t_{s} \rightarrow q t_{s}$.

See Kadell [9-11], Stembridge [15], and Zeilberger [17, 18] for some surprisingly simple proofs of certain constant term identities associated with root systems using this idea.

Let $|q|<1$ and set $(x ; q)_{m}=\prod_{i=1}^{m}\left(1-x q^{i-1}\right)$. Following [1], we set

$$
{ }_{q} f_{n}\left(a_{1}, \ldots, a_{n} ; t_{1}, \ldots, t_{n}\right)=\prod_{1 \leq i<j \leq n}\left(\frac{t_{i}}{t_{j}} ; q\right)_{a_{i}}\left(q \frac{t_{j}}{t_{i}} ; q\right)_{a_{j}} .
$$


Andrews' $q$-Dyson conjecture [1],

$$
[1]_{q} f_{n}\left(a_{1}, \ldots, a_{n} ; t_{1}, \ldots, t_{n}\right)=\frac{(q ; q)_{a_{1}+\cdots+a_{n}}}{(q ; q)_{a_{1}} \cdots(q ; q)_{a_{n}}}
$$

has been established by Zeilberger and Bressoud [19] and extended by Bressoud and Goulden [3].

In Section 2, we use the engine (1.5) of our machine to give a constant term identity which involves the weighted averages $K_{n, 0}\left(a_{1}, \ldots, a_{n}\right)$ and $K_{n, 1}\left(a_{1}, \ldots, a_{n}\right)$.

In Section 3, we continue with the engine (1.5) of our machine and give a functional equation which gives the behavior of $K_{n, m}\left(a_{1}, \ldots, a_{n}\right)$ as a function of $m$.

In Section 4, we establish the Dyson constant term identity (1.8) and then complete the proof of Theorem 1 .

In Section 5 , we outline the use of Good's proof [5] to evaluate the non-constant term coefficients $\left[t_{1} / t_{n}\right] f_{n}\left(a_{1}, \ldots, a_{n} ; t_{1}, \ldots, t_{n}\right), \quad\left[t_{1} t_{2} / t_{n-1} t_{n}\right] f_{n}\left(a_{1}, \ldots, a_{n} ; t_{1}, \ldots, t_{n}\right)$, and $\left[t_{1} t_{2} / t_{n}^{2}\right] f_{n}\left(a_{1}, \ldots, a_{n} ; t_{1}, \ldots, t_{n}\right)$. We give a conjecture with some surprising symmetries and its $q$-analogue.

\section{The machine meets Dyson}

In this section, we use the engine (1.5) of our machine to give a constant term identity which involves the weighted averages $K_{n, 0}\left(a_{1}, \ldots, a_{n}\right)$ and $K_{n, 1}\left(a_{1}, \ldots, a_{n}\right)$.

We apply the engine (1.5) of our machine by using the fact that

$$
0=\sum_{1 \leq p \neq s \leq n}[1] t_{s} \frac{\partial}{\partial t_{s}}\left(\left(1-\frac{t_{p}}{t_{s}}\right) f_{n}\left(a_{1}, \ldots, a_{n} ; t_{1}, \ldots, t_{n}\right)\right) .
$$

Observe that

$$
\begin{aligned}
s \frac{\partial}{\partial s}\left(\left(1-\frac{s}{t}\right)^{a}\left(1-\frac{t}{s}\right)^{b}\right) & =\left(\frac{-a s / t}{1-s / t}+\frac{b t / s}{1-t / s}\right)\left(1-\frac{s}{t}\right)^{a}\left(1-\frac{t}{s}\right)^{b} \\
& =\frac{a s+b t}{s-t}\left(1-\frac{s}{t}\right)^{a}\left(1-\frac{t}{s}\right)^{b} .
\end{aligned}
$$

Interchanging the roles of $s, t$ and $a, b$ in (2.2), we have

$$
t \frac{\partial}{\partial t}\left(\left(1-\frac{s}{t}\right)^{a}\left(1-\frac{t}{s}\right)^{b}\right)=\frac{a s+b t}{t-s}\left(1-\frac{s}{t}\right)^{a}\left(1-\frac{t}{s}\right)^{b} .
$$

Replacing $a$ by $a+1$ in (2.3), we obtain

$$
t \frac{\partial}{\partial t}\left(\left(1-\frac{s}{t}\right)^{a+1}\left(1-\frac{t}{s}\right)^{b}\right)=\frac{(a+1) s+b t}{t-s}\left(1-\frac{s}{t}\right)^{a+1}\left(1-\frac{t}{s}\right)^{b} .
$$

Using (2.3) and (2.4), we obtain

$$
\begin{aligned}
t_{s} \frac{\partial}{\partial t_{s}} & \left(\left(1-\frac{t_{p}}{t_{s}}\right) f_{n}\left(a_{1}, \ldots, a_{n} ; t_{1}, \ldots, t_{n}\right)\right) \\
= & \left(\frac{\left(a_{p}+1\right) t_{p}+a_{s} t_{s}}{t_{s}-t_{p}}+\sum_{v \in[1, n]-\{s, p\}} \frac{a_{s} t_{s}+a_{v} t_{v}}{t_{s}-t_{v}}\right) \\
& \times\left(1-\frac{t_{p}}{t_{s}}\right) f_{n}\left(a_{1}, \ldots, a_{n} ; t_{1}, \ldots, t_{n}\right) .
\end{aligned}
$$


Substituting (2.5) into (2.1) and rearranging the result, we obtain

$$
\begin{aligned}
0= & \sum_{1 \leq p \neq s \leq n}[1] \frac{\left(a_{p}+1\right) t_{p}+a_{s} t_{s}}{t_{s}-t_{p}}\left(1-\frac{t_{p}}{t_{s}}\right) f_{n}\left(a_{1}, \ldots, a_{n} ; t_{1}, \ldots, t_{n}\right) \\
& +\sum_{1 \leq p \neq s \leq n} \sum_{v \in[1, n]-\{p, s\}}[1] \frac{a_{s} t_{s}+a_{v} t_{v}}{t_{s}-t_{v}}\left(1-\frac{t_{p}}{t_{s}}\right) f_{n}\left(a_{1}, \ldots, a_{n} ; t_{1}, \ldots, t_{n}\right) .
\end{aligned}
$$

We now remove the denominators from the terms in the two sums on the right side of (2.6).

For the first sum on the right side of (2.6), observe that

$$
\begin{aligned}
\frac{\left(a_{p}+1\right) t_{p}+a_{s} t_{s}}{t_{s}-t_{p}}\left(1-\frac{t_{p}}{t_{s}}\right) & =\left(a_{p}+1\right) \frac{t_{p}}{t_{s}}+a_{s} \\
& =a_{p}+a_{s}+1-\left(a_{p}+1\right)\left(1-\frac{t_{p}}{t_{s}}\right) .
\end{aligned}
$$

Hence, we have

$$
\begin{aligned}
& \sum_{1 \leq p \neq s \leq n}[1] \frac{\left(a_{p}+1\right) t_{p}+a_{s} t_{s}}{t_{s}-t_{p}}\left(1-\frac{t_{p}}{t_{s}}\right) f_{n}\left(a_{1}, \ldots, a_{n} ; t_{1}, \ldots, t_{n}\right) \\
& \quad=\sum_{1 \leq p \neq s \leq n}[1]\left(a_{p}+a_{s}+1-\left(a_{p}+1\right)\left(1-\frac{t_{p}}{t_{s}}\right)\right) f_{n}\left(a_{1}, \ldots, a_{n} ; t_{1}, \ldots, t_{n}\right) .
\end{aligned}
$$

For the second sum on the right side of (2.6), we have

$$
\sum_{1 \leq p \neq s \leq n} \sum_{v \in[1, n]-\{p, s\}} h(p, s, v)=\sum_{p=1}^{n} \sum_{\substack{1 \leq s<v \leq n \\ s, v \in[1, n]-\{p\}}} h(p, s, v)+h(p, v, s),
$$

and we observe that

$$
\begin{gathered}
\frac{a_{s} t_{s}+a_{v} t_{v}}{t_{s}-t_{v}}\left(1-\frac{t_{p}}{t_{s}}\right)+\frac{a_{v} t_{v}+a_{s} t_{s}}{t_{v}-t_{s}}\left(1-\frac{t_{p}}{t_{v}}\right)=\frac{t_{p}}{t_{s} t_{v}}\left(a_{s} t_{s}+a_{v} t_{v}\right) \\
=a_{s} \frac{t_{p}}{t_{v}}+a_{v} \frac{t_{p}}{t_{s}}=a_{s}+a_{v}-a_{s}\left(1-\frac{t_{p}}{t_{v}}\right)-a_{v}\left(1-\frac{t_{p}}{t_{s}}\right) .
\end{gathered}
$$

Setting

$$
h(p, s, v)=[1] \frac{a_{s} t_{s}+a_{v} t_{v}}{t_{s}-t_{v}}\left(1-\frac{t_{p}}{t_{s}}\right) f_{n}\left(a_{1}, \ldots, a_{n} ; t_{1}, \ldots, t_{n}\right)
$$

in (2.9) and using (2.10), we obtain

$$
\begin{gathered}
\sum_{1 \leq p \neq s \leq n} \sum_{v \in[1, n]-\{p, s\}}[1] \frac{a_{s} t_{s}+a_{v} t_{v}}{t_{s}-t_{v}}\left(1-\frac{t_{p}}{t_{s}}\right) f_{n}\left(a_{1}, \ldots, a_{n} ; t_{1}, \ldots, t_{n}\right) \\
=\sum_{p=1}^{n} \sum_{\substack{1 \leq s<v \leq n \\
s, v \in[1, n]-\{p\}}}[1]\left(\frac{a_{s} t_{s}+a_{v} t_{v}}{t_{s}-t_{v}}\left(1-\frac{t_{p}}{t_{s}}\right)+\frac{a_{v} t_{v}+a_{s} t_{s}}{t_{v}-t_{s}}\left(1-\frac{t_{p}}{t_{v}}\right)\right) \\
\times f_{n}\left(a_{1}, \ldots, a_{n} ; t_{1}, \ldots, t_{n}\right)
\end{gathered}
$$




$$
\begin{gathered}
=\sum_{p=1}^{n} \sum_{\substack{1 \leq s<v \leq n \\
s, v \in[1, n]-\{p\}}}[1]\left(a_{s}+a_{v}-a_{s}\left(1-\frac{t_{p}}{t_{v}}\right)-a_{v}\left(1-\frac{t_{p}}{t_{s}}\right)\right) \\
\times f_{n}\left(a_{1}, \ldots, a_{n} ; t_{1}, \ldots, t_{n}\right) .
\end{gathered}
$$

Substituting (2.8) and (2.12) into (2.6) gives

$$
\begin{aligned}
& 0=\sum_{1 \leq p \neq s \leq n}[1]\left(a_{p}+a_{s}+1-\left(a_{p}+1\right)\left(1-\frac{t_{p}}{t_{s}}\right)\right) f_{n}\left(a_{1}, \ldots, a_{n} ; t_{1}, \ldots, t_{n}\right) \\
& +\sum_{p=1}^{n} \sum_{\substack{1 \leq s<v \leq n \\
s, v \in[1, n]-\{p\}}}[1]\left(a_{s}+a_{v}-a_{s}\left(1-\frac{t_{p}}{t_{v}}\right)-a_{v}\left(1-\frac{t_{p}}{t_{s}}\right)\right) f_{n}\left(a_{1}, \ldots, a_{n} ; t_{1}, \ldots, t_{n}\right),
\end{aligned}
$$

which we may rearrange as

$$
\begin{aligned}
& \left(\left(\sum_{1 \leq p \neq s \leq n} a_{p}+a_{s}+1\right)+\left(\sum_{p=1}^{n} \sum_{\substack{1 \leq s<v \leq n \\
s, v \in[1, n]-\{p\}}} a_{s}+a_{v}\right)\right) F_{n}\left(a_{1}, \ldots, a_{n}\right) \\
& \quad=\sum_{1 \leq p \neq s \leq n}[1]\left(a_{p}+1\right)\left(1-\frac{t_{p}}{t_{s}}\right) f_{n}\left(a_{1}, \ldots, a_{n} ; t_{1}, \ldots, t_{n}\right) \\
& \quad+\sum_{p=1}^{n} \sum_{\substack{1 \leq s<v \leq n \\
s, v \in[1, n]-\{p\}}}[1]\left(a_{s}\left(1-\frac{t_{p}}{t_{v}}\right)+a_{v}\left(1-\frac{t_{p}}{t_{s}}\right)\right) f_{n}\left(a_{1}, \ldots, a_{n} ; t_{1}, \ldots, t_{n}\right) .
\end{aligned}
$$

Using (2.9) and interchanging the roles of $s$ and $v$ in the last sum, we see that (2.14) gives

$$
\begin{aligned}
& \left(\left(\sum_{1 \leq p \neq s \leq n} a_{p}+a_{s}+1\right)+\left(\sum_{p=1}^{n} \sum_{\substack{1 \leq s<v \leq n \\
s, v \in[1, n]-\{p\}}} a_{s}+a_{v}\right)\right) F_{n}\left(a_{1}, \ldots, a_{n}\right) \\
& =\sum_{1 \leq p \neq s \leq n}[1]\left(a_{p}+1\right)\left(1-\frac{t_{p}}{t_{s}}\right) f_{n}\left(a_{1}, \ldots, a_{n} ; t_{1}, \ldots, t_{n}\right) \\
& \quad+\sum_{1 \leq p \neq s \leq n} \sum_{v \in[1, n]-\{p, s\}}[1] a_{v}\left(1-\frac{t_{p}}{t_{s}}\right) f_{n}\left(a_{1}, \ldots, a_{n} ; t_{1}, \ldots, t_{n}\right) .
\end{aligned}
$$

Observe that

$$
\begin{aligned}
& \left(\sum_{1 \leq p \neq s \leq n} a_{p}+a_{s}+1\right)+\left(\sum_{p=1}^{n} \sum_{\substack{1 \leq s<v \leq n \\
s, v \in[1, n]-\{p\}}} a_{s}+a_{v}\right) \\
& \quad=2(n-1)\left(\sum_{v=1}^{n} a_{v}\right)+n(n-1)+(n-1)(n-2)\left(\sum_{v=1}^{n} a_{v}\right) \\
& \quad=n(n-1)\left(1+\sum_{v=1}^{n} a_{v}\right) .
\end{aligned}
$$


Choose $p$ and $s$ with $1 \leq p \neq s \leq n$. Then we have

$$
a_{p}+1+\sum_{v \in[1, n]-\{p, s\}} a_{v}=1+\sum_{v \in[1, n]-\{s\}} a_{v} .
$$

Substituting (2.16) and (2.17) into (2.15), we obtain the functional equation

$$
\begin{aligned}
& n(n-1)\left(1+\sum_{v=1}^{n} a_{v}\right) F_{n}\left(a_{1}, \ldots, a_{n}\right) \\
& \quad=\sum_{1 \leq p \neq s \leq n}\left(1+\sum_{v \in[1, n]-\{s\}} a_{v}\right)[1]\left(1-\frac{t_{p}}{t_{s}}\right) f_{n}\left(a_{1}, \ldots, a_{n} ; t_{1}, \ldots, t_{n}\right) .
\end{aligned}
$$

Observe that $|M|=0$ requires that $M=\emptyset$. Setting $m=0$ in the definition (1.14), we have

$$
K_{n, 0}\left(a_{1}, \ldots, a_{n}\right)=n\left(1+\sum_{v=1}^{n} a_{v}\right) F_{n}\left(a_{1}, \ldots, a_{n}\right) .
$$

Thus, (2.18) becomes

$$
(n-1) K_{n, 0}\left(a_{1}, \ldots, a_{n}\right)=K_{n, 1}\left(a_{1}, \ldots, a_{n}\right),
$$

in agreement with Theorem 1.

\section{A functional equation involving the parameter $m$}

In this section, we continue with the engine (1.5) of our machine and give a functional equation which gives the behavior of $K_{n, m}\left(a_{1}, \ldots, a_{n}\right)$ as a function of $m$.

Let $1 \leq m \leq n-1$. We apply the engine (1.5) of our machine by using the fact that

$$
0=\sum_{1 \leq p \neq s \leq n} \sum_{\substack{M \subseteq[1, n]-p, s \\|M|=m-1}}[1] t_{s} \frac{\partial}{\partial t_{s}}\left(\left(1-\frac{t_{p}}{t_{s}}\right) \prod_{j \in M}\left(1-\frac{t_{p}}{t_{j}}\right) f_{n}\left(a_{1}, \ldots, a_{n} ; t_{1}, \ldots, t_{n}\right)\right) .
$$

Observe that the case $m=1$ of (3.1) is (2.1) which gave the functional equation (2.20).

Let $M \subseteq[1, n]-p, s$ with $|M|=m-1$. Using (2.3) and (2.4), we obtain

$$
\begin{aligned}
t_{s} \frac{\partial}{\partial t_{s}} & \left(\left(1-\frac{t_{p}}{t_{s}}\right) \prod_{j \in M}\left(1-\frac{t_{p}}{t_{j}}\right) f_{n}\left(a_{1}, \ldots, a_{n} ; t_{1}, \ldots, t_{n}\right)\right) \\
= & \frac{\left(a_{p}+1\right) t_{p}+a_{s} t_{s}}{t_{s}-t_{p}}+\sum_{v \in M} \frac{a_{s} t_{s}+a_{v} t_{v}}{t_{s}-t_{v}}+\sum_{v \in[1, n]-M-\{p, s\}} \frac{a_{s} t_{s}+a_{v} t_{v}}{t_{s}-t_{v}} \\
& \times\left(1-\frac{t_{p}}{t_{s}}\right) \prod_{j \in M}\left(1-\frac{t_{p}}{t_{j}}\right) f_{n}\left(a_{1}, \ldots, a_{n} ; t_{1}, \ldots, t_{n}\right) .
\end{aligned}
$$

Substituting (3.2) into (3.1) and rearranging the result, we obtain

$$
\begin{aligned}
0=\sum_{1 \leq p \neq s \leq n} \sum_{\substack{M \subseteq[1, n]-p, s \\
|M|=m-1}}[1] \frac{\left(a_{p}+1\right) t_{p}+a_{s} t_{s}}{t_{s}-t_{p}}\left(1-\frac{t_{p}}{t_{s}}\right) \\
\quad \times \prod_{j \in M}\left(1-\frac{t_{p}}{t_{j}}\right) f_{n}\left(a_{1}, \ldots, a_{n} ; t_{1}, \ldots, t_{n}\right)+
\end{aligned}
$$




$$
\begin{aligned}
& +\sum_{\substack { 1 \leq p \neq s \leq n \\
\begin{subarray}{c}{M \subseteq[1, n]-p, s \\
|M|=m-1{ 1 \leq p \neq s \leq n \\
\begin{subarray} { c } { M \subseteq [ 1 , n ] - p , s \\
| M | = m - 1 } }\end{subarray}} \sum_{v \in M}[1] \frac{a_{s} t_{s}+a_{v} t_{v}}{t_{s}-t_{v}}\left(1-\frac{t_{p}}{t_{s}}\right) \\
& +\prod_{j \in M}\left(1-\frac{t_{p}}{t_{j}}\right) f_{n}\left(a_{1}, \ldots, a_{n} ; t_{1}, \ldots, t_{n}\right) \\
& +\sum_{1 \leq p \neq s \leq n} \sum_{\substack{M \subseteq[1, n]-p, s \\
|M|=m-1}} \sum_{v \in[1, n]-M-p, s}[1] \frac{a_{s} t_{s}+a_{v} t_{v}}{t_{s}-t_{v}}\left(1-\frac{t_{p}}{t_{s}}\right) \\
& \quad \times \prod_{j \in M}\left(1-\frac{t_{p}}{t_{j}}\right) f_{n}\left(a_{1}, \ldots, a_{n} ; t_{1}, \ldots, t_{n}\right) .
\end{aligned}
$$

We now remove the denominators from the terms in the three sums on the right side of (3.3).

Using (2.7), the first sum on the right side of (3.3) becomes

$$
\begin{gathered}
\sum_{1 \leq p \neq s \leq n} \sum_{\substack{M \subseteq[1, n]-p, s \\
|M|=m-1}}[1] \frac{\left(a_{p}+1\right) t_{p}+a_{s} t_{s}}{t_{s}-t_{p}}\left(1-\frac{t_{p}}{t_{s}}\right) \\
\times \prod_{j \in M}\left(1-\frac{t_{p}}{t_{j}}\right) f_{n}\left(a_{1}, \ldots, a_{n} ; t_{1}, \ldots, t_{n}\right) \\
=\sum_{1 \leq p \neq s \leq n} \sum_{\substack{M \subseteq[1, n]-p, s \\
|M|=m-1}}[1]\left(a_{p}+a_{s}+1-\left(a_{p}+1\right)\left(1-\frac{t_{p}}{t_{s}}\right)\right) \\
\times \prod_{j \in M}\left(1-\frac{t_{p}}{t_{j}}\right) f_{n}\left(a_{1}, \ldots, a_{n} ; t_{1}, \ldots, t_{n}\right) .
\end{gathered}
$$

Observe that when $m=1$, the condition $|M|=m-1=0$ implies that $M=\emptyset$ and the second sum in (3.3) is zero. Thus, for the second sum on the right side of (3.3), we may assume that $2 \leq m \leq n-1$. We have

$$
\begin{aligned}
& \sum_{1 \leq p \neq s \leq n} \sum_{\substack{M \subseteq[1, n]-p, s \\
|M|=m-1}} \sum_{v \in M} h_{M}(p, s, v) \\
& \quad=\sum_{p=1}^{n} \sum_{\substack{1 \leq s<v \leq n \\
s, v \in[1, n]-\{p\}}} \sum_{\substack{M \subseteq[1, n]-\{p, s, v\} \\
|M|=m-2}} h_{M \cup\{v\}}(p, s, v)+h_{M \cup\{s\}}(p, v, s) .
\end{aligned}
$$

Observe that the function

$$
h_{M}(p, s, v)=[1] \frac{a_{s} t_{s}+a_{v} t_{v}}{t_{s}-t_{v}}\left(1-\frac{t_{p}}{t_{s}}\right) \prod_{j \in M}\left(1-\frac{t_{p}}{t_{j}}\right) f_{n}\left(a_{1}, \ldots, a_{n} ; t_{1}, \ldots, t_{n}\right)
$$

satisfies

$$
\begin{gathered}
M \subseteq[1, n]-\{p, s, v\}, \quad 1 \leq s<v \leq n, \quad s, v \in[1, n]-\{p\} \\
\Longrightarrow \quad h_{M \cup\{s\}}(p, v, s)=-h_{M \cup\{v\}}(p, s, v)
\end{gathered}
$$


since the factor $\left(1-t_{p} / t_{s}\right)\left(1-t_{p} / t_{v}\right)$ appears in both $h_{M \cup\{s\}}(p, v, s)$ and $h_{M \cup\{v\}}$ $(p, s, v)$. Thus, (3.5) gives

$$
\begin{gathered}
0=\sum_{1 \leq p \neq s \leq n} \sum_{\substack{M \subseteq[1, n]-\{p, s\} \\
|M|=m-1}} \sum_{v \in M}[1] \frac{a_{s} t_{s}+a_{v} t_{v}}{t_{s}-t_{v}}\left(1-\frac{t_{p}}{t_{s}}\right) \\
\times \prod_{j \in M}\left(1-\frac{t_{p}}{t_{j}}\right) f_{n}\left(a_{1}, \ldots, a_{n} ; t_{1}, \ldots, t_{n}\right) .
\end{gathered}
$$

For the third sum on the right side of (3.3), we have

$$
\begin{gathered}
\sum_{1 \leq p \neq s \leq n} \sum_{\substack{M \subseteq[1, n]-\{p, s\} \\
|M|=m-1}} \sum_{v \in[1, n]-M-\{p, s\}} h_{M}(p, s, v) \\
=\sum_{p=1}^{n} \sum_{\substack{1 \leq s<v \leq n \\
s, v \in[1, n]-\{p\}}} \sum_{\substack{M \subseteq[1, n]-\{p, s, v\} \\
|M|=m-1}} h_{M}(p, s, v)+h_{M}(p, v, s) .
\end{gathered}
$$

Using (2.10), we see that (3.9) gives

$$
\begin{aligned}
& \sum_{1 \leq p \neq s \leq n} \sum_{\substack{M \subseteq[1, n]-\{p, s\} \\
|M|=m-1}} \sum_{v \in[1, n]-M-\{p, s\}}[1] \frac{a_{s} t_{s}+a_{v} t_{v}}{t_{s}-t_{v}}\left(1-\frac{t_{p}}{t_{s}}\right) \\
& \times \prod_{j \in M}\left(1-\frac{t_{p}}{t_{j}}\right) f_{n}\left(a_{1}, \ldots, a_{n} ; t_{1}, \ldots, t_{n}\right) \\
& =\sum_{p=1}^{n} \sum_{\substack{1 \leq s<v \leq n \\
s, v \in[1, n]-\{p\}}} \sum_{\substack{M \subseteq[1, n]-\{p, s, v\} \\
|M|=m-1}}[1]\left(\frac{a_{s} t_{s}+a_{v} t_{v}}{t_{s}-t_{v}}\left(1-\frac{t_{p}}{t_{s}}\right)+\frac{a_{v} t_{v}+a_{s} t_{s}}{t_{v}-t_{s}}\left(1-\frac{t_{p}}{t_{v}}\right)\right) \\
& \times \prod_{j \in M}\left(1-\frac{t_{p}}{t_{j}}\right) f_{n}\left(a_{1}, \ldots, a_{n} ; t_{1}, \ldots, t_{n}\right) \\
& =\sum_{p=1}^{n} \sum_{\substack{1 \leq s<v \leq n \\
s, v \in[1, n]-\{p\}}} \sum_{\substack{M \subseteq[1, n]-\{p, s, v\} \\
|M|=m-1}}[1]\left(a_{s}+a_{v}-a_{s}\left(1-\frac{t_{p}}{t_{v}}\right)-a_{v}\left(1-\frac{t_{p}}{t_{s}}\right)\right) \\
& \times \prod_{j \in M}\left(1-\frac{t_{p}}{t_{j}}\right) f_{n}\left(a_{1}, \ldots, a_{n} ; t_{1}, \ldots, t_{n}\right) .
\end{aligned}
$$

Substituting (3.4), (3.8), and (3.10) into (3.3), we obtain

$$
\begin{aligned}
0= & \sum_{1 \leq p \neq s \leq n} \sum_{\substack{M \subseteq[1, n]-\{p, s\} \\
|M|=m-1}}[1]\left(a_{p}+a_{s}+1-\left(a_{p}+1\right)\left(1-\frac{t_{p}}{t_{s}}\right)\right) \\
& \times \prod_{j \in M}\left(1-\frac{t_{p}}{t_{j}}\right) f_{n}\left(a_{1}, \ldots, a_{n} ; t_{1}, \ldots, t_{n}\right) \\
& +\sum_{p=1}^{n} \sum_{\substack{1 \leq s<v \leq n \\
s, v \in[1, n]-\{p\}}} \sum_{\substack{M \subseteq[1, n]-\{p, s, v\} \\
|M|=m-1}}[1]\left(a_{s}+a_{v}-a_{s}\left(1-\frac{t_{p}}{t_{v}}\right)-a_{v}\left(1-\frac{t_{p}}{t_{s}}\right)\right) \times
\end{aligned}
$$




$$
\times \prod_{j \in M}\left(1-\frac{t_{p}}{t_{j}}\right) f_{n}\left(a_{1}, \ldots, a_{n} ; t_{1}, \ldots, t_{n}\right),
$$

which we may rearrange as

$$
\begin{aligned}
& \sum_{1 \leq p \neq s \leq n} \sum_{\substack{M \subseteq[1, n]-\{p, s\} \\
|M|=m-1}}[1]\left(a_{p}+a_{s}+1\right) \prod_{j \in M}\left(1-\frac{t_{p}}{t_{j}}\right) f_{n}\left(a_{1}, \ldots, a_{n} ; t_{1}, \ldots, t_{n}\right) \\
& +\sum_{p=1}^{n} \sum_{\substack{1 \leq s<v \leq n \\
s, v \in[1, n]-\{p\}}} \sum_{\substack{M \subseteq[1, n]-\{p, s, v\} \\
|M|=m-1}}[1]\left(a_{s}+a_{v}\right) \prod_{j \in M}\left(1-\frac{t_{p}}{t_{j}}\right) f_{n}\left(a_{1}, \ldots, a_{n} ; t_{1}, \ldots, t_{n}\right) \\
& =\sum_{1 \leq p \neq s \leq n} \sum_{\substack{M \subseteq[1, n]-\{p, s\} \\
|M|=m-1}}[1]\left(a_{p}+1\right)\left(1-\frac{t_{p}}{t_{s}}\right) \prod_{j \in M}\left(1-\frac{t_{p}}{t_{j}}\right) f_{n}\left(a_{1}, \ldots, a_{n} ; t_{1}, \ldots, t_{n}\right) \\
& +\sum_{p=1}^{n} \sum_{\substack{1 \leq s<v \leq n \\
s, v \in[1, n]-\{p\}}}[1]\left(a_{s}\left(1-\frac{t_{p}}{t_{v}}\right)+a_{v}\left(1-\frac{t_{p}}{t_{s}}\right)\right) \\
& \quad \times \prod_{\substack{M \in M|=m-\{p, s, v\}\\
| M \mid=m-1}}\left(1-\frac{t_{p}}{t_{j}}\right) f_{f_{n}\left(a_{1}, \ldots, a_{n} ; t_{1}, \ldots, t_{n}\right) .}
\end{aligned}
$$

Using (3.9) and interchanging the roles of $s$ and $v$ in the last sum, we see that (3.12) gives

$$
\begin{aligned}
& \sum_{1 \leq p \neq s \leq n} \sum_{\substack{M \subseteq[1, n]-\{p, s\} \\
|M|=m-1}}[1]\left(a_{p}+a_{s}+1\right) \prod_{j \in M}\left(1-\frac{t_{p}}{t_{j}}\right) f_{n}\left(a_{1}, \ldots, a_{n} ; t_{1}, \ldots, t_{n}\right) \\
& +\sum_{p=1}^{n} \sum_{\substack{1 \leq s<v \leq n \\
s, v \in[1, n]-\{p\}}} \sum_{\substack{M \subseteq[1, n]-\{p, s, v\} \\
|M|=m-1}}[1]\left(a_{s}+a_{v}\right) \prod_{j \in M}\left(1-\frac{t_{p}}{t_{j}}\right) f_{n}\left(a_{1}, \ldots, a_{n} ; t_{1}, \ldots, t_{n}\right) \\
& =\sum_{1 \leq p \neq s \leq n} \sum_{\substack{M \subseteq[1, n]-\{p, s\} \\
|M|=m-1}}[1]\left(a_{p}+1\right)\left(1-\frac{t_{p}}{t_{s}}\right) \prod_{j \in M}\left(1-\frac{t_{p}}{t_{j}}\right) f_{n}\left(a_{1}, \ldots, a_{n} ; t_{1}, \ldots, t_{n}\right) \\
& +\sum_{\substack{1 \leq p \neq s \leq n \\
M \subseteq[1, n]-\{p, s\} \\
\mid M \in[1, n]-M-\{p, s\}}}[1] a_{v}\left(1-\frac{t_{p}}{t_{s}}\right) \\
& \quad \times \prod_{j \in M}\left(1-\frac{t_{p}}{t_{j}}\right) f_{n}\left(a_{1}, \ldots, a_{n} ; t_{1}, \ldots, t_{n}\right) .
\end{aligned}
$$

Observe that

$$
\begin{aligned}
& \sum_{1 \leq p \neq s \leq n} \sum_{\substack{M \subseteq[1, n]-\{p, s\} \\
|M|=m-1}}[1]\left(a_{p}+a_{s}+1\right) \prod_{j \in M}\left(1-\frac{t_{p}}{t_{j}}\right) f_{n}\left(a_{1}, \ldots, a_{n} ; t_{1}, \ldots, t_{n}\right) \\
& \quad=\sum_{p=1}^{n} \sum_{\substack{M \subseteq[1, n]-\{p\} \\
|M|=m-1}}\left((n-m)\left(a_{p}+1\right)+\sum_{v \in[1, n]-M-\{p\}} a_{v}\right) \times
\end{aligned}
$$




$$
\times \prod_{j \in M}\left(1-\frac{t_{p}}{t_{j}}\right) f_{n}\left(a_{1}, \ldots, a_{n} ; t_{1}, \ldots, t_{n}\right)
$$

and

$$
\begin{aligned}
\sum_{p=1}^{n} \sum_{\substack{1 \leq s<v \leq n \\
s, v \in[1, n]-\{p\}}} \sum_{\substack{M \subseteq[1, n]-\{p, s, v\} \\
|M|=m-1}}[1]\left(a_{s}+a_{v}\right) \prod_{j \in M}\left(1-\frac{t_{p}}{t_{j}}\right) f_{n}\left(a_{1}, \ldots, a_{n} ; t_{1}, \ldots, t_{n}\right) \\
=(n-m-1) \sum_{p=1}^{n} \sum_{\substack{M \subseteq[1, n]-\{p\} \\
|M|=m-1}}\left(\sum_{v \in[1, n]-M-\{p\}} a_{v}\right) \\
\quad \times[1] \prod_{j \in M}\left(1-\frac{t_{p}}{t_{j}}\right) f_{n}\left(a_{1}, \ldots, a_{n} ; t_{1}, \ldots, t_{n}\right) .
\end{aligned}
$$

Observe that the sum of (3.14) and (3.15) is given by

$$
\begin{aligned}
& \sum_{1 \leq p \neq s \leq n} \sum_{\substack{M \subseteq[1, n]-\{p, s\} \\
|M|=m-1}}[1]\left(a_{p}+a_{s}+1\right) \prod_{j \in M}\left(1-\frac{t_{p}}{t_{j}}\right) f_{n}\left(a_{1}, \ldots, a_{n} ; t_{1}, \ldots, t_{n}\right) \\
& +\sum_{p=1}^{n} \sum_{\substack{1 \leq s<v \leq n \\
s, v \in[1, n]-\{p\}}} \sum_{\substack{M \subseteq[1, n]-\{p, s, v\} \\
|M|=m-1}}[1]\left(a_{s}+a_{v}\right) \prod_{j \in M}\left(1-\frac{t_{p}}{t_{j}}\right) f_{n}\left(a_{1}, \ldots, a_{n} ; t_{1}, \ldots, t_{n}\right) \\
& =(n-m) \sum_{p=1}^{n} \sum_{\substack{M \subseteq[1, n]-\{p\} \\
|M|=m-1}}\left(1+\sum_{v \in[1, n]-M} a_{v}\right) \\
& \quad \times[1] \prod_{j \in M}\left(1-\frac{t_{p}}{t_{j}}\right) f_{n}\left(a_{1}, \ldots, a_{n} ; t_{1}, \ldots, t_{n}\right) .
\end{aligned}
$$

We also have

$$
\begin{gathered}
\sum_{1 \leq p \neq s \leq n} \sum_{\substack{M \subseteq[1, n]-\{p, s\} \\
|M|=m-1}}[1]\left(a_{p}+1\right)\left(1-\frac{t_{p}}{t_{s}}\right) \prod_{j \in M}\left(1-\frac{t_{p}}{t_{j}}\right) f_{n}\left(a_{1}, \ldots, a_{n} ; t_{1}, \ldots, t_{n}\right) \\
=m \sum_{p=1}^{n} \sum_{\substack{M \subseteq[1, n]-\{p\} \\
|M|=m}}\left(a_{p}+1\right)[1] \prod_{j \in M}\left(1-\frac{t_{p}}{t_{j}}\right) f_{n}\left(a_{1}, \ldots, a_{n} ; t_{1}, \ldots, t_{n}\right)
\end{gathered}
$$

and

$$
\begin{aligned}
& \sum_{1 \leq p \neq s \leq n} \sum_{\substack{M \subseteq[1, n]-\{p, s\} \\
|M|=m-1}} \sum_{v \in[1, n]-M-\{p, s\}}[1] a_{v}\left(1-\frac{t_{p}}{t_{s}}\right) \\
& \times \prod_{j \in M}\left(1-\frac{t_{p}}{t_{j}}\right) f_{n}\left(a_{1}, \ldots, a_{n} ; t_{1}, \ldots, t_{n}\right) \\
& =m \sum_{p=1}^{n} \sum_{\substack{M \subseteq[1, n]-\{p\} \\
|M|=m}}\left(\sum_{v \in[1, n]-M-\{p\}} a_{v}\right)[1] \prod_{j \in M}\left(1-\frac{t_{p}}{t_{j}}\right) f_{n}\left(a_{1}, \ldots, a_{n} ; t_{1}, \ldots, t_{n}\right) .
\end{aligned}
$$


Observe that the sum of (3.17) and (3.18) is given by

$$
\begin{aligned}
& \sum_{1 \leq p \neq s \leq n} \sum_{\substack{M \subseteq[1, n]-\{p, s\} \\
|M|=m-1}}[1]\left(a_{p}+1\right)\left(1-\frac{t_{p}}{t_{s}}\right) \prod_{j \in M}\left(1-\frac{t_{p}}{t_{j}}\right) f_{n}\left(a_{1}, \ldots, a_{n} ; t_{1}, \ldots, t_{n}\right) \\
& +\sum_{1 \leq p \neq s \leq n} \sum_{\substack{M \subseteq[1, n]-\{p, s\} \\
|M|=m-1}} \sum_{v \in[1, n]-M-\{p, s\}}[1] a_{v}\left(1-\frac{t_{p}}{t_{s}}\right) \\
& \quad \times \prod_{j \in M}\left(1-\frac{t_{p}}{t_{j}}\right) f_{n}\left(a_{1}, \ldots, a_{n} ; t_{1}, \ldots, t_{n}\right) \\
& =m \sum_{p=1}^{n} \sum_{\substack{M \subseteq[1, n]-\{p\} \\
|M|=m}}\left(1+\sum_{v \in[1, n]-M} a_{v}\right)[1] \prod_{j \in M}\left(1-\frac{t_{p}}{t_{j}}\right) f_{n}\left(a_{1}, \ldots, a_{n} ; t_{1}, \ldots, t_{n}\right) .
\end{aligned}
$$

Combining our results (3.14)-(3.19), we see that (3.13) becomes

$$
\begin{gathered}
(n-m) \sum_{p=1}^{n} \sum_{\substack{M \subseteq[1, n]-\{p\} \\
|M|=m-1}}\left(1+\sum_{v \in[1, n]-M} a_{v}\right)[1] \prod_{j \in M}\left(1-\frac{t_{p}}{t_{j}}\right) f_{n}\left(a_{1}, \ldots, a_{n} ; t_{1}, \ldots, t_{n}\right) \\
=m \sum_{p=1}^{n} \sum_{\substack{M \subseteq[1, n]-\{p\} \\
|M|=m}}\left(1+\sum_{v \in[1, n]-M} a_{v}\right)[1] \prod_{j \in M}\left(1-\frac{t_{p}}{t_{j}}\right) f_{n}\left(a_{1}, \ldots, a_{n} ; t_{1}, \ldots, t_{n}\right) .
\end{gathered}
$$

Comparing with the definition (1.14), we see that the functional equation (3.20) is given by

$$
(n-m) K_{n, m-1}\left(a_{1}, \ldots, a_{n}\right)=m K_{n, m}\left(a_{1}, \ldots, a_{n}\right), \quad 1 \leq m \leq n-1 .
$$

Observe that this agrees with (2.20) when $m=1$.

\section{A proof of Theorem 1}

In this section, we establish the Dyson constant term identity (1.8) and then complete the proof of Theorem 1.

Observe that Theorem 1 is trivially true for $m=n$ since

$$
K_{n, n}\left(a_{1}, \ldots, a_{n}\right)=k_{n, n}\left(a_{1}, \ldots, a_{n} ; t_{1}, \ldots, t_{n}\right)=n\left(\begin{array}{c}
n-1 \\
n
\end{array}\right)=0 .
$$

We may rearrange (3.21) as

$$
K_{n, m}\left(a_{1}, \ldots, a_{n}\right)=\frac{(n-m)}{m} K_{n, m-1}\left(a_{1}, \ldots, a_{n}\right) .
$$

Using (4.2), we see by induction on $m$ that

$$
\begin{aligned}
K_{n, m}\left(a_{1}, \ldots, a_{n}\right) & =\prod_{j=1}^{m} \frac{(n-j)}{j} K_{n, 0}\left(a_{1}, \ldots, a_{n}\right) \\
& =\left(\begin{array}{c}
n-1 \\
m
\end{array}\right) K_{n, 0}\left(a_{1}, \ldots, a_{n}\right),
\end{aligned}
$$


in agreement with Theorem 1.

Setting $m=n-1$ in (4.3) gives

$$
K_{n, n-1}\left(a_{1}, \ldots, a_{n}\right)=K_{n, 0}\left(a_{1}, \ldots, a_{n}\right) .
$$

Observe from the definition (1.14) that

$$
\begin{aligned}
K_{n, n-1}\left(a_{1}, \ldots, a_{n}\right) & =[1] k_{n, n-1}\left(a_{1}, \ldots, a_{n} ; t_{1}, \ldots, t_{n}\right) \\
& =\sum_{p=1}^{n}\left(1+a_{p}\right)[1] \prod_{j \in[1, n]-\{p\}}\left(1-\frac{t_{p}}{t_{j}}\right) f_{n}\left(a_{1}, \ldots, a_{n} ; t_{1}, \ldots, t_{n}\right) \\
& =\sum_{p=1}^{n}\left(1+a_{p}\right) F_{n}\left(a_{1}, \ldots, a_{p-1}, a_{p}+1, a_{p+1}, \ldots, a_{n}\right)
\end{aligned}
$$

Substituting (4.5) and (2.19) into (4.4) gives

$$
\sum_{p=1}^{n}\left(1+a_{p}\right) F_{n}\left(a_{1}, \ldots, a_{p-1}, a_{p}+1, a_{p+1}, \ldots, a_{n}\right)=n\left(1+\sum_{v=1}^{n} a_{v}\right) F_{n}\left(a_{1}, \ldots, a_{n}\right) .
$$

We now use the functional equation (3.21) to prove the Dyson constant term identity (1.8) where $n$ is a positive integer and $a_{1}, \ldots, a_{n}$ are nonnegative integers.

We proceed by induction on $n$ and the minimum

$$
z=\min \left(a_{1}, \ldots, a_{n}\right)
$$

of the parameters $a_{1}, \ldots, a_{n}$.

Observe that when $a_{m}=0$ for $1 \leq m \leq n$, the variable $t_{m}$ does not occur to a positive power in any of the terms in the expansion of $f_{n}\left(a_{1}, \ldots, a_{n} ; t_{1}, \ldots, t_{n}\right)$. Thus, we have the boundary condition

$$
\begin{aligned}
& F_{n}\left(a_{1}, \ldots, a_{m-1}, 0, a_{m+1}, \ldots, a_{n}\right) \\
& \quad=F_{n-1}\left(a_{1}, \ldots, a_{m-1}, a_{m+1}, \ldots, a_{n}\right), \quad 1 \leq m \leq n,
\end{aligned}
$$

which using our induction assumption on $n$ establishes the Dyson constant term identity (1.8) when $a_{m}=0$ for some $m$ with $1 \leq m \leq n$.

We now assume that $a_{1}, \ldots, a_{n}$ are positive integers. We let $a_{u}=z$ where $1 \leq u \leq n$ and we have $z \geq 1$.

Replace $a_{1}, \ldots, a_{n}$ in (4.6) by $\alpha_{1}, \ldots, \alpha_{n}$ where $\alpha_{u}=z-1$ and $\alpha_{i}=a_{i}$ for $1 \leq i \leq n$, $i \neq u$. We may rearrange the result as

$$
\begin{aligned}
& a_{u} F_{n}\left(a_{1}, \ldots, a_{n}\right)=n\left(\sum_{v=1}^{n} a_{v}\right) F_{n}\left(\alpha_{1}, \ldots, \alpha_{n}\right) \\
& \quad-\sum_{\substack{m=1 \\
m \neq u}}^{n}\left(1+a_{m}\right) F_{n}\left(\alpha_{1}, \ldots, \alpha_{m-1}, \alpha_{m}+1, \alpha_{m+1}, \ldots, \alpha_{n}\right) .
\end{aligned}
$$

Observe that the multinomial coefficient $\left(a_{1}+\cdots+a_{n}\right) ! / a_{1} ! \cdots a_{n}$ ! satisfies (4.6). Each term in the sum on the left side of (4.6) equals $1 / n$ times the right side of (4.6). Thus, the multinomial coefficient also satisfies (4.9).

Observe that

$$
\min \left(\alpha_{1}, \ldots, \alpha_{n}\right)=z-1
$$


and

$$
1 \leq m \leq n \text { and } m \neq u \Longrightarrow \min \left(\alpha_{1}, \ldots, \alpha_{m-1}, \alpha_{m}+1, \alpha_{m+1}, \ldots, \alpha_{n}\right)=z-1 .
$$

Thus, we see by our induction assumption on $z$ that all of the constant terms on the right side of (4.9) are given by the Dyson constant term identity (1.8). This completes our induction on $z$ and establishes the Dyson constant term identity (1.8).

Theorem 1 then follows using (2.19) and (4.3).

\section{A conjecture and its $q$-analogue}

In this section, we outline the use of Good's proof [5] to evaluate the non constant term coefficients $\left[t_{1} / t_{n}\right] f_{n}\left(a_{1}, \ldots, a_{n} ; t_{1}, \ldots, t_{n}\right),\left[t_{1} t_{2} / t_{n-1} t_{n}\right] f_{n}\left(a_{1}, \ldots, a_{n} ; t_{1}, \ldots, t_{n}\right)$ and $\left[t_{1} t_{2} / t_{n}^{2}\right] f_{n}\left(a_{1}, \ldots, a_{n} ; t_{1}, \ldots, t_{n}\right)$. We give a conjecture with some surprising symmetries and its $q$-analogue.

Extracting the coefficient of $t_{1} / t_{n}$ from (1.11), we see that the coefficient

$$
\mathcal{F}_{n}\left(a_{1}, \ldots, a_{n}\right)=\left[t_{1} / t_{n}\right] f_{n}\left(a_{1}, \ldots, a_{n} ; t_{1}, \ldots, t_{n}\right)
$$

satisfies the functional equation (1.12). Observe that it also satisfies the boundary conditions

$$
\begin{aligned}
& \mathcal{F}_{n}\left(0, a_{2}, \ldots, a_{n}\right)=0 \\
& \mathcal{F}_{n}\left(a_{1}, \ldots, a_{r-1}, 0, a_{r+1}, \ldots, a_{n}\right)=\mathcal{F}_{n-1}\left(a_{1}, \ldots, a_{r-1}, a_{r+1}, \ldots, a_{n}\right) \\
& 2 \leq r \leq n-1
\end{aligned}
$$

and

$$
\begin{aligned}
\mathcal{F}_{n}\left(a_{1}, \ldots, a_{n-1}, 0\right)= & -a_{1} F_{n-1}\left(a_{1}, \ldots, a_{n-1}\right) \\
& -\left(a_{2}+\cdots+a_{n-1}\right) \mathcal{F}_{n-1}\left(a_{1}, \ldots, a_{n-1}\right) .
\end{aligned}
$$

The reader may readily use Good's proof [5] to obtain

$$
\left(1+\sum_{v=2}^{n} a_{v}\right) \mathcal{F}_{n}\left(a_{1}, \ldots, a_{n}\right)=-a_{1} \frac{\left(a_{1}+\cdots+a_{n}\right) !}{a_{1} ! \cdots a_{n} !} .
$$

Observe that the coefficient $\mathcal{F}_{n}\left(a_{1}, \ldots, a_{n}\right)$ is symmetric in $a_{2}, \ldots, a_{n}$.

Let $1 \leq p \neq s \leq n$. Taking the Dyson constant term identity (1.9) minus (5.1) and using the symmetry (1.17) with the transpositions $\pi=(p, n)$ and $\pi=(1, s)$, we obtain

$$
\begin{aligned}
(1+ & \left.\sum_{v \in[1, n]-\{s\}} a_{v}\right)[1]\left(1-\frac{t_{p}}{t_{s}}\right) f_{n}\left(a_{1}, \ldots, a_{n} ; t_{1}, \ldots, t_{n}\right) \\
& =\left(1+\sum_{v=1}^{n} a_{v}\right) \frac{\left(a_{1}+\cdots+a_{n}\right) !}{a_{1} ! \cdots a_{n} !} .
\end{aligned}
$$

Observe that (5.6) states that the contribution of each term on the right side of (2.18) to the constant term $K_{n, 1}\left(a_{1}, \ldots, a_{n}\right)$ is independent of $p$ and $s$.

The reader may consult [12] for the details of using Good's proof [5] to establish the non-constant term coefficient identity

$$
\left[t_{1} t_{2} / t_{n-1} t_{n}\right] f_{n}\left(a_{1}, \ldots, a_{n} ; t_{1}, \ldots, t_{n}\right)=\left[t_{1} t_{2} / t_{n}^{2}\right] f_{n}\left(a_{1}, \ldots, a_{n} ; t_{1}, \ldots, t_{n}\right)
$$




$$
\begin{aligned}
= & \left.\frac{a_{1}}{\left(1+\sum_{v=2}^{n} a_{v}\right)} \frac{a_{2}}{\left(1+\sum_{v=3}^{n} a_{v}\right)}+\frac{a_{2}}{\left(1+a_{1}+\sum_{v=3}^{n} a_{v}\right)} \frac{a_{1}}{\left(1+\sum_{v=3}^{n} a_{v}\right)}\right) \\
& \times \frac{\left(a_{1}+\cdots+a_{n}\right) !}{a_{1} ! \cdots a_{n} !} .
\end{aligned}
$$

Observe that this common coefficient is symmetric in $a_{1}, a_{2}$ and in $a_{3}, \ldots, a_{n}$.

Let $3 \leq p_{1}, p_{2} \leq n$. Using the constant term identities (5.1) and (5.7), their symmetries, and some tedious computation, we obtain

$$
\begin{aligned}
(1+ & \left.\sum_{v=3}^{n} a_{v}\right)[1]\left(1-\frac{t_{p_{1}}}{t_{1}}\right)\left(1-\frac{t_{p_{2}}}{t_{2}}\right) f_{n}\left(a_{1}, \ldots, a_{n} ; t_{1}, \ldots, t_{n}\right) \\
& =\left(1+\sum_{v=1}^{n} a_{v}\right) \frac{\left(a_{1}+\cdots+a_{n}\right) !}{a_{1} ! \cdots a_{n} !} .
\end{aligned}
$$

Observe that (5.8) states that the contribution of each term on the right side of the definition (1.14) to the constant term $K_{n, 2}\left(a_{1}, \ldots, a_{n}\right)$ is independent of $p$ and the subset $M$. Moreover, we may let $p$ be a function whose range is a subset of $[1, n]-M$.

This leads us to make the following conjecture which provides a refinement of Theorem 1.

Conjecture 2. Let $M \subseteq[1, n],|M|=m, 0 \leq m \leq n-1$, and $\left\{p_{s} \mid s \in M\right\} \cap M=\emptyset$. Then we have

$$
\begin{aligned}
(1+ & \left.\sum_{v \in[1, n]-M} a_{v}\right)[1] \prod_{s \in M}\left(1-\frac{t_{p_{s}}}{t_{s}}\right) f_{n}\left(a_{1}, \ldots, a_{n} ; t_{1}, \ldots, t_{n}\right) \\
& =\left(1+\sum_{v=1}^{n} a_{v}\right) \frac{\left(a_{1}+\cdots+a_{n}\right) !}{a_{1} ! \cdots a_{n} !} .
\end{aligned}
$$

Conjecture 2 states that the contribution of each term on the right side of the definition (1.14) to the constant term $K_{n, m}\left(a_{1}, \ldots, a_{n}\right)$ is independent of $p$ and the subset $M$. Moreover, we may let $p$ be a function whose range is a subset of $[1, n]-M$. That is, we may replace the variable $t_{p}$ in the numerator of the extra factors in the definition (1.14) by $t_{p_{s}}$ where $t_{p_{s}} \in[1, n]-M$ for all $s \in M$.

It is well known (see $[1,7]$ ) that the case $n=2$ of $(1.21)$

$$
\text { [1] }\left(\frac{s}{t} ; q\right)_{a}\left(q \frac{t}{s} ; q\right)_{b}=\frac{(q ; q)_{a+b}}{(q ; q)_{a}(q ; q)_{b}}
$$

is equivalent to the $q$-binomial theorem. Observe that we may interpret the symmetry of (5.10) in $a$ and $b$ as stating that if we add one to the subscript $a$ or $b$ and multiply by $\left(1-q^{a+1}\right)$ or $\left(1-q^{b+1}\right)$, respectively, then we obtain the same constant term. In particular, the presence or absence of $q$ in the argument $s / t$ or $q t / s$ does not affect the result.

This leads us to make the following conjecture which provides a $q$-analogue of Conjecture 2.

Conjecture 3. Let $M \subseteq[1, n],|M|=m, 0 \leq m \leq n-1$, and $\left\{p_{s} \mid s \in M\right\} \cap M=\emptyset$. Then we have

$$
\left(1-q^{1+\sum_{v \in[1, n]-M} a_{v}}\right)[1] \prod_{1 \leq i<j \leq n}\left(\frac{t_{i}}{t_{j}} ; q\right)_{a_{i}+\chi\left(j \in M \text { and } i=p_{j}\right)}\left(q \frac{t_{j}}{t_{i}} ; q\right)_{a_{j}+\chi\left(i \in M \text { and } j=p_{i}\right)}
$$




$$
=\left(1-q^{1+\sum_{v=1}^{n} a_{v}}\right) \frac{(q ; q)_{a_{1}+\cdots+a_{n}}}{(q ; q)_{a_{1}} \cdots(q ; q)_{a_{n}}} .
$$

Observe that the right side of (5.11) is independent of the function $p$ and the subset $M$. In [7], we observed the elementary symmetry

$$
{ }_{q} f_{n}\left(a_{2}, \ldots, a_{n}, a_{1} ; t_{2}, \ldots, t_{n}, q t_{1}\right)={ }_{q} f_{n}\left(a_{1}, \ldots, a_{n} ; t_{1}, \ldots, t_{n}\right),
$$

which extends (1.17) for long cycles in $S_{n}$. As happened in [8], (5.12) allows us to compute the partial $q$-derivatives required by the $q$-engine (1.19). Since the case $a_{1}=\cdots=a_{n}=k$ of $(5.12)$ is central to the simple proof in [10], we may ask if there is a simple proof of the Zeilberger-Bressoud theorem (1.21) using the symmetry (5.12) and the fact that the constant term is unchanged by the substitution $t_{s} \rightarrow q t_{s}$ where $1 \leq s \leq n$.

Acknowledgment. Research supported by National Science Foundation grant DMS 9212416.

\section{References}

1. G. E. Andrews, Problems and prospects for basic hypergeometric functions. In: Theory and Application of Special Functions (Ed. R. A. Askey), Academic Press, New York, 1975.

2. K. Aomoto, Jacobi polynomials associated with Selberg's integral, SIAM J. Math. Anal. 18 (1987), 545-549.

3. D. M. Bressoud and I. P. Goulden, Constant term identities extending the $q-D y s o n$ theorem, Trans. Amer. Math. Soc. 291 (1985), 203-228.

4. F. J. Dyson, Statistical theory of the energy levels of complex systems I, J. Math. Phys. 3 (1962), 140-156.

5. I. J. Good, Short proof of a conjecture of Dyson, J. Math. Phys. 11 (1970), 1884.

6. J. Gunson, Proof of a conjecture by Dyson in the statistical theory of energy levels, J. Math. Phys. 3 (1962), 752-753.

7. K. W. J. Kadell, $A$ proof of Andrews' $q$-Dyson conjecture for $n=4$, Trans. Amer. Math. Soc. 290 (1985), 127-144.

8. - A proof of Askey's conjectured q-analogue of Selberg's integral and a conjecture of Morris, SIAM J. Math. Anal. 19 (1988), 969-986.

9. (1994), No. 516.

10. 166 (1994), 167-181.

11. _ , A simple proof of an Aomoto-type extension of Gustafson's Askey-Wilson Selberg qintegral, Methods Appl. Anal., 5 (1998), 125-142.

12. The Dyson polynomials, preprint.

13. W. G. Morris, II, Constant term identities for finite and affine root systems: conjectures and theorems, Ph.D. dissertation, University of Wisconsin-Madison, January 1982.

14. A. Selberg, Bemerkninger om et multipelt integral, Norsk Mat. Tidsskr. 26 (1943), 71-78.

15. J. Stembridge, $A$ short proof of Macdonald's conjecture for the root system of type $A$, Proc. Amer. Math. Soc. 102 (1988), 777-786.

16. K. Wilson, Proof of a conjecture by Dyson, J. Math. Phys. 3 (1962), 1040-1043.

17. D. Zeilberger, A unified approach to Macdonald's root-system conjectures, SIAM J. Math. Anal. 19 (1988), 987-1013.

18. _ A Stembridge-Stanton style elementary proof of the Habsieger-Kadell q-Morris identity, Discrete Math. 79 (1989/90), 313-322.

19. D. Zeilberger and D. M. Bressoud, A proof of Andrews' $q$-Dyson conjecture, Discrete Math. 54 (1985), 201-224.

Department of Mathematics, Arizona State University, Tempe, AZ 85287-1804, USA 